\title{
Vacuum Condensate, Geometric Phase, Unruh Effect, and Temperature Measurement
}

\author{
Antonio Capolupo and Giuseppe Vitiello \\ Dipartimento di Fisica E.R. Caianiello, Università di Salerno, and INFN Gruppo Collegato di Salerno, SA, 84084 Fisciano, Italy \\ Correspondence should be addressed to Antonio Capolupo; capolupo@sa.infn.it
}

Received 15 April 2015; Revised 7 June 2015; Accepted 22 June 2015

Academic Editor: Piero Nicolini

Copyright (C) 2015 A. Capolupo and G. Vitiello. This is an open access article distributed under the Creative Commons Attribution License, which permits unrestricted use, distribution, and reproduction in any medium, provided the original work is properly cited. The publication of this article was funded by SCOAP

\begin{abstract}
In our previous work the possibility to use the Aharonov-Anandan invariant as a tool in the analysis of disparate systems has been shown, including Hawking and Unruh effects, as well as graphene physics and thermal states. We show that the vacuum condensation, characterizing such systems, is also related with geometric phases and we analyze the properties of the geometric phase of systems represented by mixed state and undergoing a nonunitary evolution. In particular, we consider twolevel atoms accelerated by an external potential and interacting with a thermal state. We propose the realization of Mach-Zehnder interferometers which can prove the existence of the Unruh effect and can allow very precise measurements of temperature.
\end{abstract}

\section{Introduction}

It is hard to observe phenomena like Unruh [1], Hawking [2], and Parker effects [3, 4]. However it has been shown [5] that in such phenomena as well as in all the systems where the vacuum condensates are generated [6-12], the AharonovAnandan invariant (AAI) [13] is produced in their evolution. Moreover, it has been shown $[14,15]$ that such an invariant is related to the geometric phase [16-37]. This fact suggests that in all the above phenomena, in which the presence of AAIs has been revealed [5], an associated geometric phase also appears. Then, the study of such a phase could open a new way to the detection of effects elusive to the detection.

Here, instead of using AAI, which is experimentally difficult to be observed, we use geometric phases to study the Unruh effect and the possibility to perform very precise measurement of temperature. Geometric phases have been detected in many physical systems [38-42] and have been also related to CPT symmetry [43] and SUSY violation in thermal states [44].

In the present paper, we focus our attention on systems of atoms accelerated in an electromagnetic field and atoms interacting with thermal states, so that we study geometric phases for mixed states in nonunitary, noncyclic evolution. Different approaches exist in dealing with geometric phases and mixed states [31-37]; in this paper we mostly follow the Wang and Liu approach [30].

In our treatment, besides the relation between boson condensation and geometric phase, we obtain two novel results, one concerning atoms accelerated in an electromagnetic field and the Unruh effect and the other one exhibiting the possibility to perform very precise measurement of temperature. In the first case, a detectable difference of the geometric phases appears between the accelerated and the inertial atoms. Such a phase difference is due only to the Unruh effect. In the second case, the difference between geometric phases produced by atoms interacting with two different thermal states allows to determine the temperature of a sample once the temperature of the other one (assumed as reference temperature) is known.

The idea of using geometric phases and invariants to probe the Unruh effect $[5,45,46]$ and to build a thermometer $[5,47]$ has been already presented in previous works (the study of dynamical phase to have precise estimation of the temperature has been proposed in [48]). In this paper we consider a realistic scheme for experimental implementations and we study the geometric phase defined in [30], which generalize the Berry phase (used in $[46,47]$ ), to the case of quantum open systems. The use of the phase presented in [30] allows to consider time intervals arbitrary small 
(we do not need to consider cyclic evolutions and their related period). In a similar way the discussion reported in [30] allows to consider the cases of very low transition frequencies, as well as spontaneous emission rates characterizing fine and hyperfine atomic structures. Indeed, in the short time intervals which we consider, the number of spontaneously emitted particle is negligible and the systems are quasistable.

The Berry phase for mixed states cannot be used in the study of the systems which we consider. One reason is that our systems do not undergo cyclic evolutions because of their interaction with the environment [30]. Even in the approximation of a quasicyclicity of mixed states, the number of spontaneously emitted particles becomes not negligible in time intervals of the order of $T=2 \pi / \omega_{0}$ (with $\omega_{0}$ atomic transition frequency). Thus the analysis of the geometric phase loses meaning.

Our treatment offers other improvements, which cannot be obtained by using the Berry phase. For example, in the present treatment, by considering the fine and hyperfine atomic structure, we have access to lower acceleration regimes, which in turn improves the detection of Unruh effect and permits very precise temperature measurements.

We consider the structure of the atomic levels of ${ }^{85} R b$, ${ }^{87} \mathrm{Rb}$, and ${ }^{133} \mathrm{Cs}$. We suggest that a Mach-Zehnder interferometer of $4 \mathrm{~cm}$, where the hyperfine level of these atoms is considered, may reveal the Unruh effect with accelerations of order of $10^{16} \mathrm{~m} / \mathrm{s}^{2}$ and that a similar device could be used also for very precise temperature measurements.

In Section 2 we link together AAI, geometric phase, and vacuum condensates and introduce the geometric phase for mixed states in nonunitary evolution. In Section 3 we analyze the geometric phase for a two-level atom undergoing a nonunitary evolution and in Sections 4 and 5 we study the realization of a Mach-Zehnder interferometer to reveal the Unruh effect and to build a very precise thermometer. Section 6 is devoted to the conclusions.

\section{Geometric Phase}

The geometric phase for pure states can be expressed as [15]

$$
\begin{aligned}
\Phi(\Gamma) & =\int_{\Gamma} \sqrt{d D^{2}-d S^{2}} \\
& =\int_{\Gamma}\left[\left\langle\frac{\phi}{\|\phi\|}\left|i \frac{d}{d t}-\dot{\psi}(t)\right| \frac{\phi}{\|\phi\|}\right\rangle\right] d t,
\end{aligned}
$$

which also establishes the relation with the AAI. Here $d D^{2}$ denotes the infinitesimal "reference distance" in the projective Hilbert space $P$ and is given by

$$
\begin{gathered}
d D^{2}=\left[\left\langle\frac{d}{d t}\left(\frac{\phi}{\|\phi\|}\right) \mid \frac{d}{d t}\left(\frac{\phi}{\|\phi\|}\right)\right\rangle+\dot{\psi}^{2}\right. \\
\left.-2 i \dot{\psi}\left\langle\frac{\phi}{\|\phi\|} \mid \frac{d}{d t}\left(\frac{\phi}{\|\phi\|}\right)\right\rangle\right] d t^{2},
\end{gathered}
$$

with $\psi(t)=(i / 2) \ln [\langle\phi(0) \mid \phi(t)\rangle /\langle\phi(t) \mid \phi(0)\rangle]$. In (1), $d S^{2}$ is the Fubini-Study metric given by

$$
\begin{aligned}
d S^{2} & =\left(\left\langle\frac{d}{d t}\left(\frac{\phi}{\|\phi\|}\right) \mid \frac{d}{d t}\left(\frac{\phi}{\|\phi\|}\right)\right\rangle\right. \\
- & {\left.\left[i\left\langle\frac{\phi}{\|\phi\|} \mid \frac{d}{d t}\left(\frac{\phi}{\|\phi\|}\right)\right\rangle\right]^{2}\right) d t^{2} . }
\end{aligned}
$$

The AAI is the total length of the path measured using the Fubini-Study metric $S$ expressed as $S=(2 / \hbar) \int_{0}^{t} \Delta E\left(t^{\prime}\right) d t^{\prime}$ [13]. In the above formulas $|\phi(t)\rangle$ is a nonstationary state with energy uncertainty $\Delta E(t)$ given by $\Delta E^{2}(t)=\left\langle\phi(t)\left|H^{2}\right| \phi(t)\right\rangle-$ $(\langle\phi(t)|H| \phi(t)\rangle)^{2}$. Since the length of the path " $S$ " is the minimum length measured by the "reference distance" function " $D$ " $[14,15]$, the presence of the AAI in the evolution of a system implies the presence of the " $D$ " invariant and consequentially the existence of the geometric phase (1). As already mentioned in the Introduction, the AAI is produced in the evolution of systems with vacuum condensates [5] (see the Appendix); then (1) suggests that vacuum condensation is also related with geometric phases.

Vacuum fluctuations may produce particle creation from vacuum or vacuum condensate in disparate ways [1-12]. Then the geometric phase may provide the possibility to study the properties of many systems and could be used to detect effects so far elusive to the observations, ranging from the Unruh effect to Casimir effect, including other phenomena of quantum field theory (QFT) in curved background.

As said, in the present paper we focus on the Unruh effect and on thermal states, studying the possible realization of a quantum thermometer. In our analysis we consider quantum open systems and analyze the geometric phase for mixed states in nonunitary, noncyclic evolution. In particular, we use the Wang and Liu approach [30] and the geometric phase defined as

$$
\begin{aligned}
\Phi_{g} & =\frac{1}{N} \\
& \sum_{k=1}^{N} \arg \left[\sqrt{\left[1+b r\left(t_{0}\right) \varphi_{k}\right]\left[1+b r(t) \varphi_{k}\right]}\left\langle\varphi_{k}\left(t_{0}\right) \mid \varphi_{k}(t)\right\rangle\right] \\
& -\frac{1}{N} \Im \sum_{k=1}^{N} \int_{t_{0}}^{t}\left[1+b r\left(t^{\prime}\right) \varphi_{k}\right]\left\langle\varphi_{k}\left(t^{\prime}\right)\left|\frac{\partial}{\partial t^{\prime}}\right| \varphi_{k}\left(t^{\prime}\right)\right\rangle d t^{\prime},
\end{aligned}
$$

where the first term of the right side represents the total phase, while the second term is the dynamic one. In (4), $r(t)$ is the Block radius, satisfying the condition $0 \leq r(t) \leq 1$, $b=\sqrt{N(N-1) / 2}$, and $\varphi_{k}$ are the eigenvalues of the operator $\sum_{i=1}^{N^{2}-1} n_{i}(t) \lambda_{i}$ (with $\lambda_{i}$ traceless and hermitian $N \times N$ operators and $\left.n_{i}(t)=(N / 2 b) \operatorname{Tr}\left[\lambda_{i} \rho(t)\right]\right)$ which permits to write $\rho(t)$ as $\rho(t)=(1 / N)\left(1+b \sum_{i=1}^{N^{2}-1} n_{i}(t) \lambda_{i}\right)$. Moreover, $\left|\varphi_{k}(t)\right\rangle$ and $\lambda_{k}(t) \equiv\left[1+b r(t) \varphi_{k}\right] / N$ are eigenstates and eigenvalues of the density matrix $\rho$ of $N$-level mixed states, respectively:

$$
\rho\left|\varphi_{k}(t)\right\rangle=\frac{\left(1+b r(t) \varphi_{k}\right)}{N}\left|\varphi_{k}(t)\right\rangle .
$$


Then the geometric phase (4) can be expressed also as

$$
\begin{aligned}
\Phi_{g}= & \sum_{k=1}^{N} \arg \left[\sqrt{\lambda_{k}\left(t_{0}\right) \lambda_{k}(t)}\left\langle\varphi_{k}\left(t_{0}\right) \mid \varphi_{k}(t)\right\rangle\right] \\
& -\Im \sum_{k=1}^{N} \int_{t_{0}}^{t} \lambda_{k}\left(t^{\prime}\right)\left\langle\varphi_{k}\left(t^{\prime}\right)\left|\frac{\partial}{\partial t^{\prime}}\right| \varphi_{k}\left(t^{\prime}\right)\right\rangle d t^{\prime} .
\end{aligned}
$$

We consider a two-level open system. In this case, $N=2$, $b=1$, and the density matrix $\rho$ is expressed in terms of Pauli matrices $\sigma_{i}$ as

$$
\rho(t)=\frac{1}{2}\left(1+\sum_{i=1}^{3} n_{i}(t) \sigma_{i}\right)
$$

with $n_{i}=\operatorname{Tr}\left(\rho \sigma_{i}\right)$ and radius of Block sphere given by $r(t)=$ $\sqrt{n_{1}^{2}+n_{2}^{2}+n_{3}^{2}}$. Explicitly, one has

$$
\begin{aligned}
& n_{1}=\rho_{12}+\rho_{21}, \\
& n_{2}=i\left(\rho_{12}-\rho_{21}\right), \\
& n_{3}=\rho_{11}-\rho_{22} .
\end{aligned}
$$

Introducing the angles such as

$$
\begin{aligned}
\theta & =\cos ^{-1}\left(\frac{n_{3}}{r}\right), \\
\phi & =\tan \left(\frac{n_{2}}{n_{1}}\right),
\end{aligned}
$$

the eigenvectors $\left|\varphi_{1}(t)\right\rangle$ and $\left|\varphi_{2}(t)\right\rangle$ (apart from overall phase factors which do not contribute to $\Phi_{g}$ ) are

$$
\begin{aligned}
& \left|\varphi_{1}(t)\right\rangle=\left(\begin{array}{c}
\cos \frac{\theta(t)}{2} \\
e^{i \phi(t)} \sin \frac{\theta(t)}{2}
\end{array}\right), \\
& \left|\varphi_{2}(t)\right\rangle=\left(\begin{array}{c}
\sin \frac{\theta(t)}{2} \\
-e^{i \phi(t)} \cos \frac{\theta(t)}{2}
\end{array}\right),
\end{aligned}
$$

and the eigenvalues of the operator $\sum_{i=1}^{3} n_{i}(t) \sigma_{i}$ are $\varphi_{1}=1$ and $\varphi_{2}=-1$. Therefore $\lambda_{1}(t)=(1 / 2)[1+r(t)]$ and $\lambda_{2}=$ $(1 / 2)[1-r(t)]$.

\section{Geometric Phase and Two-Level Atoms}

We consider the interaction of an atom with vacuum modes of the electromagnetic field in the multipolar scheme [49] and treat the atom as an open system with a nonunitary evolution in the reservoir of the electromagnetic field. The Hamiltonian of the atom and the reservoir is

$$
H=\frac{\hbar}{2} \omega_{0} \sigma_{3}+H_{F}-\sum_{m n} \mu_{m n} \cdot \mathbf{E}(x(t)) \sigma_{m n}
$$

where $\omega_{0}$ is the energy level spacing of the atom, $\sigma_{3}$ is the Pauli matrix, $H_{F}$ is the electromagnetic field Hamiltonian, $\mu_{m n}$ is the matrix element of the dipole momentum operator connecting single-particle states $u_{n}$ and $u_{n^{\prime}}$ (see [49]), $\sigma_{m n}=$ $\sigma_{m} \sigma_{n}$, and $\mathbf{E}$ is the strength of the electric field. Denoting by $|0\rangle$ and $\rho(0)$ the vacuum and the initial reduced density matrix of the atom, respectively, we analyze the evolution of the total density matrix $\rho_{\text {tot }}=\rho(0) \otimes|0\rangle\langle 0|$, in the frame of the atom. We assume a weak interaction between atom and field; then the evolution can be written as $[50,51]$

$$
\begin{aligned}
\frac{\partial \rho(\tau)}{\partial \tau}= & -\frac{i}{\hbar}\left[H_{\mathrm{eff}}, \rho(\tau)\right] \\
& +\frac{1}{2} \sum_{i, j=1}^{3} a_{i j}\left(2 \sigma_{j} \rho \sigma_{i}-\sigma_{i} \sigma_{j} \rho-\rho \sigma_{i} \sigma_{j}\right) .
\end{aligned}
$$

Here $\tau$ is the proper time and $a_{i j}$ are the coefficients of the Kossakowski matrix

$$
a_{i j}=\Sigma \delta_{i j}-i \Upsilon \epsilon_{i j k} \delta_{k 3}-\Sigma \delta_{i 3} \delta_{j 3}
$$

with

$$
\begin{array}{r}
\Sigma=\frac{1}{4}\left[G\left(\omega_{0}\right)+G\left(-\omega_{0}\right)\right], \\
\Upsilon=\frac{1}{4}\left[G\left(\omega_{0}\right)-G\left(-\omega_{0}\right)\right], \\
G(\omega)=\int_{-\infty}^{\infty} d \tau e^{i \omega \tau} G^{+}(x(\tau)) .
\end{array}
$$

$G(\omega)$ is the Fourier transform of $G^{+}(x-y)$ :

$$
\begin{aligned}
& G^{+}(x-y) \\
& \quad=\frac{e^{2}}{\hbar^{2}} \sum_{i, j=1}^{3}\left\langle+\left|r_{i}\right|-\right\rangle\left\langle-\left|r_{j}\right|+\right\rangle\left\langle 0\left|E_{i}(x) E_{j}(x)\right| 0\right\rangle .
\end{aligned}
$$

$H_{\text {eff }}$ is the effective hamiltonian, $H_{\text {eff }}=(\hbar / 2) \Omega \sigma_{3}$, where $\Omega$ is the renormalized energy level spacing containing the Lamb shift terms. Such terms can be neglected in the computation of the geometric phase; thus we approximate the effective level spacing of the atoms $\Omega$ with the atomic transition frequency $\omega_{0}$; that is $\Omega \sim \omega_{0}$.

By writing $\rho(\tau)$ in terms of Pauli matrices, as in (7), and considering the initial state of the atom,

$$
|\psi(0)\rangle=\cos \left(\frac{\theta}{2}\right)|+\rangle+\sin \left(\frac{\theta}{2}\right)|-\rangle
$$


with $\theta \equiv \theta(0)$, one can derive the reduced density matrix

$\rho(\tau)[5,45]$

$$
\rho(\tau)=\left(\begin{array}{cc}
e^{-4 \Sigma \tau} \cos ^{2}\left(\frac{\theta}{2}\right)+\frac{\Upsilon-\Sigma}{2 \Sigma}\left(e^{-4 \Sigma \tau}-1\right) & \frac{1}{2} e^{-2 \Sigma \tau-i \Omega \tau} \sin \theta \\
\frac{1}{2} e^{-2 \Sigma \tau+i \Omega \tau} \sin \theta & 1-e^{-4 \Sigma \tau} \cos ^{2}\left(\frac{\theta}{2}\right)-\frac{\Upsilon-\Sigma}{2 \Sigma}\left(e^{-4 \Sigma \tau}-1\right)
\end{array}\right) .
$$

Denoting by $\xi(\tau)$ and $\chi(\tau)$ the following quantities

$$
\begin{aligned}
\xi(\tau) & =\sqrt{\chi^{2}+e^{-4 \Sigma \tau} \sin ^{2} \theta}, \\
\chi(\tau) & =e^{-4 \Sigma \tau} \cos \theta+\frac{\Upsilon}{\Sigma}\left(e^{-4 \Sigma \tau}-1\right),
\end{aligned}
$$

the matrix (18) can be written as

$$
\rho(\tau)=\frac{1}{2}\left(\begin{array}{cc}
\chi+1 & e^{-i \Omega \tau} \sqrt{\xi^{2}-\chi^{2}} \\
e^{i \Omega \tau} \sqrt{\xi^{2}-\chi^{2}} & 1-\chi
\end{array}\right) .
$$

Then, (8), the Block radius and the angles defined in (9) are given by

$$
\begin{aligned}
& n_{1}(\tau)=\sqrt{\xi^{2}-\chi^{2}} \cos (\Omega \tau), \\
& n_{2}(\tau)=\sqrt{\xi^{2}-\chi^{2}} \sin (\Omega \tau), \\
& n_{3}(\tau)=\chi, \\
& r(\tau)=\xi, \\
& \theta(\tau)=\cos ^{-1} \frac{\chi}{\xi}, \\
& \phi(\tau)=\Omega \tau,
\end{aligned}
$$

respectively. The eigenvalues of $\rho(\tau)$ are

$$
\lambda_{ \pm}=\frac{1}{2}(1 \pm r)=\frac{1}{2}(1 \pm \xi),
$$

and the corresponding eigenvectors are

$$
\begin{aligned}
& \left|\phi_{+}(\tau)\right\rangle=\cos \left(\frac{\theta(\tau)}{2}\right)|+\rangle+\sin \left(\frac{\theta(\tau)}{2}\right) e^{i \Omega \tau}|-\rangle, \\
& \left|\phi_{-}(\tau)\right\rangle=\sin \left(\frac{\theta(\tau)}{2}\right)|+\rangle-\cos \left(\frac{\theta(\tau)}{2}\right) e^{i \Omega \tau}|-\rangle .
\end{aligned}
$$

We consider the initial time $t_{0}=0$. Being $\lambda_{-}(0)=0$, the phase (6) becomes

$$
\begin{aligned}
\Phi_{g}(t)= & \arg \left[\sqrt{\lambda_{+}(0) \lambda_{+}(t)}\left\langle\phi_{+}(0) \mid \phi_{+}(t)\right\rangle\right] \\
& -\mathfrak{J} \int_{t_{0}}^{t} \lambda_{-}\left(t^{\prime}\right)\left\langle\phi_{-}\left(t^{\prime}\right)\left|\frac{\partial}{\partial t^{\prime}}\right| \phi_{-}\left(t^{\prime}\right)\right\rangle d t^{\prime} \\
& -\mathfrak{I} \int_{t_{0}}^{t} \lambda_{+}\left(t^{\prime}\right)\left\langle\phi_{+}\left(t^{\prime}\right)\left|\frac{\partial}{\partial t^{\prime}}\right| \phi_{+}\left(t^{\prime}\right)\right\rangle d t^{\prime},
\end{aligned}
$$

which reduces to

$$
\begin{aligned}
\Phi_{g}(t)= & \arg \left[\cos \frac{\theta}{2} \cos \frac{\theta(t)}{2}+\sin \frac{\theta}{2} \sin \frac{\theta(t)}{2} e^{i \Omega t}\right] \\
& -\frac{\Omega}{2} \int_{0}^{t}[1-\xi(\tau) \cos \theta(\tau)] d \tau,
\end{aligned}
$$

with $\theta \equiv \theta(0)$.

In the next sections we consider the use of (29) in the detection of Unruh effect and in the building of a quantum thermometer.

\section{Unruh Effect}

For an accelerated observer the ground state of an inertial system appears at a nonzero temperature depending on the acceleration of the observer. Such a phenomenon is called the Unruh effect. It has not yet been detected. Recently it has been shown that geometric phases and invariants could allow its detection in table top experiments $[5,45,46]$.

Here we show that the realization of an interferometer is possible, in which paths of slightly different lengths can be chosen in order to let the geometric phase be dominating over the relative dynamical phase. We compute the geometric phase (29) for the two-level system in the presence of an acceleration and in the inertial case. The atom interaction with the electromagnetic field itself produces a geometric phase; however, the difference between the two phases is due only to the atom acceleration and then to the Unruh effect, since the accelerated system sees the Minkowski vacuum as a thermal Rindler vacuum.

A two-level atom uniformly accelerated in the $x$ direction with acceleration $a$, through Minkowski spacetime, is conveniently described with Rindler coordinates $x(\tau)=$ $\left(c^{2} / a\right) \cosh (a \tau / c), t(\tau)=(c / a) \sinh (a \tau / c)$. For this system, the field correlation function is given by $[5,45]$

$$
\begin{aligned}
G^{+} & \left(x, x^{\prime}\right) \\
\quad= & \frac{e^{2}|\langle-|\mathbf{r}|+\rangle|^{2}}{16 \pi^{2} \varepsilon_{0} \hbar c^{7}} \frac{a^{4}}{\sinh ^{4}\left[(a / 2 c)\left(\tau-\tau^{\prime}-i \varepsilon\right)\right]},
\end{aligned}
$$

and its Fourier transform is

$$
\begin{aligned}
& G(\omega) \\
& \quad=\frac{\omega^{3} e^{2}|\langle-|\mathbf{r}|+\rangle|^{2}}{6 \pi \varepsilon_{0} \hbar c^{3}}\left(1+\frac{a^{2}}{c^{2} \omega^{2}}\right)\left(1+\operatorname{coth} \frac{\pi c \omega}{a}\right) .
\end{aligned}
$$


Then, the coefficients $\Sigma$ and $\Upsilon$ in (14) become $[5,45]$

$$
\begin{aligned}
& \Sigma_{a}=\frac{\gamma_{0}}{4}\left(1+\frac{a^{2}}{c^{2} \omega_{0}^{2}}\right) \frac{e^{2 \pi c \omega_{0} / a}+1}{e^{2 \pi c \omega_{0} / a}-1} \\
& \Upsilon_{a}=\frac{\gamma_{0}}{4}\left(1+\frac{a^{2}}{c^{2} \omega_{0}^{2}}\right)
\end{aligned}
$$

where $\gamma_{0}$ is the spontaneous emission rate and $\omega_{0}$ is the atomic transition frequency. The function $\sin (\theta(t) / 2)=$ $\pm \sqrt{(1 / 2)(1-\chi(t) / \xi(t))}$ in (29) becomes

$$
\begin{aligned}
& \sin \frac{\theta_{a}(t)}{2} \\
& \quad= \pm \sqrt{\frac{1}{2}-\frac{R_{a}-R_{a} e^{4 \Sigma_{a} t}+\cos \theta}{2 \sqrt{e^{4 \Sigma_{a} t} \sin ^{2} \theta+\left(R_{a}-R_{a} e^{4 \Sigma_{a} t}+\cos \theta\right)^{2}}}}
\end{aligned}
$$

and similar for $\cos \left(\theta_{a}(t) / 2\right)$. Here $R_{a}=\Upsilon_{a} / \Sigma_{a}$.

For an inertial atom, $a=0$, the geometric phase $\Phi_{a=0}$ assumes the identical expression of (29), with $\sin \left(\theta_{a}(t) / 2\right)$, $\cos \left(\theta_{a}(t) / 2\right)$, and $\cos \theta_{a}(t)$ replaced by $\sin \left(\theta_{a=0}(t) / 2\right)$, $\cos \left(\theta_{a=0}(t) / 2\right)$, and $\cos \theta_{a=0}(t)$ in which the coefficients $\Sigma_{a}$, $\Upsilon_{a}, R_{a}$ are replaced by $\Sigma_{a=0}=\Upsilon_{a=0}=\gamma_{0} / 4$, with $\gamma_{0}$ spontaneous emission rate, and $R_{a=0}=1$, respectively.

The phase difference between the accelerated and inertial atoms, $\Delta \Phi_{U}(t)=\Phi_{a}(t)-\Phi_{a=0}(t)$, gives the geometric phase in terms of the acceleration of the atom. The value of $\Delta \Phi_{U}(t)$ depends on the ratios $a /\left(c \omega_{0}\right)$ and $\gamma_{0} / \omega_{0}$ and on the time interval $t$. Indeed $\Delta \Phi_{U}(t)$ increases for values of the acceleration $a$ which approach $c \omega_{0}$; that is $a \sim c \omega_{0}$. Moreover, $\Delta \Phi_{U}(t)$ is detectable when $\gamma_{0} / \omega_{0}>10^{-5}$. Therefore a crucial role is played by the choice of the atomic systems used in the interferometer. Moreover, $\Delta \Phi_{U}(t)$ depends on $\theta(0)$; it reaches the maximum value for $\theta(0)=\pi / 2$. We consider an initial state with angle $\theta(0) \sim \pi / 2$ and, in order to decrease the value of the acceleration, we consider the hyperfine level structure of different atoms.

In Figure 1 we plot the difference of geometric phase $\Delta \Phi_{U}$ as function of the acceleration $a$ for different systems.

The (blue) dashed line in the inset of the figure is obtained considering the energy splitting between the levels $F=1$ and $F=2$ of the ground state $5^{2} S_{1 / 2}$ of ${ }^{85} R b(\mathbf{F}=\mathbf{J}+\mathbf{I}$ is the total atomic angular momentum, with $\mathbf{J}$ total electron angular momentum and I total nuclear angular momentum). For this system one has $\omega_{0}=3.035 \mathrm{GHz}$, and we considered the $D_{1}$ transition for which $\gamma_{0}=36.129 \mathrm{MHz}$ [52]. A similar plot is obtained if we consider the $D_{2}$ transition for which $\gamma_{0}=38.117 \mathrm{MHz}$ [52]. We derive the (red) dot dashed line in the inset by studying the energy splitting between the levels $F=1$ and $F=2$ of the $5^{2} S_{1 / 2}$ line of ${ }^{87} R b$. In this case, $\omega_{0}=6.843 \mathrm{GHz}$ and we consider the $D_{1}$ transition with $\gamma_{0}=$ $36.129 \mathrm{MHz}$ [53]. Similar plot can be derived in the case of the $D_{2}$ transition with $\gamma_{0}=38.117 \mathrm{MHz}$ [53]. Such plots show that a phase $\Delta \Phi_{U} \sim 10^{-4} \pi$ is obtained for accelerations of order of $10^{17} \mathrm{~m} / \mathrm{s}^{2}$ and the times $t$ of order of $t \sim 1 / \omega_{0}$, (inset

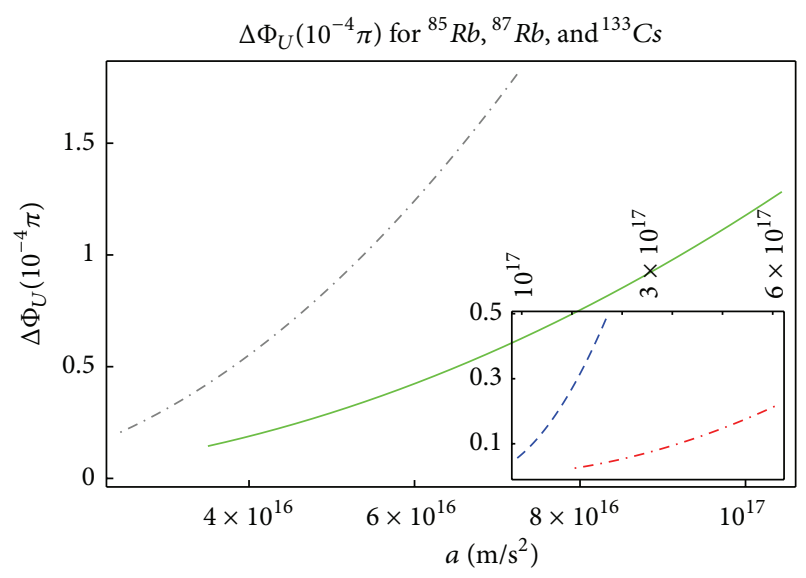

Figure 1: Plots of $\Delta \Phi_{U}$ as a function of the atom acceleration $a$, for time intervals $t \simeq 1 / \omega_{0}$ and the splitting between the hyperfine energy levels: main pictures: (gray) dot dashed line: ${ }^{87} R b, 5^{2} P_{1 / 2}$ line, splitting for $F=1 \rightarrow F=2$ transition $\left(\omega_{0}=814.5 \mathrm{MHz}\right.$, $\left.\gamma_{0}=36.129 \mathrm{MHz}[53]\right)$; (green) solid line: ${ }^{133} \mathrm{Cs}, 6{ }^{2} P_{1 / 2}$ line, splitting of the between the $F=3$ and $F=4$ levels $\left(\omega_{0}=1167.68 \mathrm{MHz}\right.$, $\left.\gamma_{0}=28.743 \mathrm{MHz}[54]\right)$. Pictures in the inset: (blue) dashed line: ${ }^{85} R b, 5^{2} S_{1 / 2}$ line, energy splitting between the levels $F=1$ and $F=2\left(\omega_{0}=3.035 \mathrm{GHz}, \gamma_{0}=36.129 \mathrm{MHz}\right.$ for $D_{1}$ transition, $\gamma_{0}=38.117 \mathrm{MHz}$ for $D_{2}$ transition [52]); (red) dot dashed line: ${ }^{87} R b, 5^{2} S_{1 / 2}$ line, energy splitting between the levels $F=1$ and $F=2\left(\omega_{0}=6.843 \mathrm{GHz}, \gamma_{0}=36.129 \mathrm{MHz}\right.$ for $D_{1}$ transition, $\gamma_{0}=38.117 \mathrm{MHz}$ for $\mathrm{D}_{2}$ transition [53]).

of Figure 1). For such time interval, the speed of the atoms is of order of $(0.2-0.3) c$ and the spontaneous emission can be neglected, since $N\left(t \sim 1 / \omega_{0}\right) \sim 0.99 N(0)$. The values of the phases obtained are accessible with the current technology.

Better results can be also obtained by considering other splitting between the levels of the ${ }^{87} R b$, and the $6^{2} P_{1 / 2}$ energy splitting between the levels of the ${ }^{133} \mathrm{Cs}$, as shown in the main pictures of Figure 1. Here, the (gray) dot dashed line represents $\Delta \Phi_{U}$ for the $5^{2} P_{1 / 2}$ energy splitting between the $F=1$ and $F=2$ levels of the ${ }^{87} \mathrm{Rb}$. In this case, $\omega_{0}=$ 814.5 MHz and $\gamma_{0}=36.129 \mathrm{MHz}$ [53]. The (green) solid line is achieved by considering the $6^{2} P_{1 / 2}$ line splitting between the $F=3$ and $F=4$ levels of ${ }^{133} \mathrm{Cs}$. For this system, $\omega_{0}=$ $1167.68 \mathrm{MHz}$ and $\gamma_{0}=28.743 \mathrm{MHz}$ [54]. In these cases $\Delta \Phi_{U} \sim 10^{-4} \pi$ can be achieved for accelerations of order of $10^{16} \mathrm{~m} / \mathrm{s}^{2}$ and speeds of order of $(0.2-0.3) c$, as shown in the main plots of Figure 1 and one has $N\left(t \sim 1 / \omega_{0}\right) \sim 0.98 N(0)$.

We now analyze the characteristic of a Mach-Zehnder interferometer able to reveal the geometric phase (28) related to the Unruh effect. The geometric phase can be detected when the dynamical phase is negligible compared with the geometric one. The total phase, for the accelerated and for the inertial atoms, is given in terms of the geometric phase $\Phi_{g}$ by the formula

$$
\Phi_{\text {tot }}(t)=\Phi_{g}(t)+\frac{\Omega}{2} \int_{0}^{t}[1-\xi(\tau) \cos \theta(\tau)] d \tau,
$$

where the second term on the right side is the dynamical phase. 
We note that the dynamic phases can be made negligible compared to the geometric ones, if the branches of the interferometer are built in order that the two dynamical phases are almost equal; that is

$$
\begin{aligned}
\delta= & \frac{\Omega}{2}\left[\int_{0}^{t^{\prime}}\left[1-\xi_{a}(\tau) \cos \theta_{a}(\tau)\right] d \tau\right. \\
& \left.-\int_{0}^{t}\left[1-\xi_{a=0}(\tau) \cos \theta_{a=0}(\tau)\right] d \tau\right] \ll \Delta \Phi_{U} .
\end{aligned}
$$

In this case the difference of total phases $\Delta \Phi_{\text {tot }}$ detected in the cross point of the interferometer corresponds almost completely to the difference of geometric phases $\Delta \Phi$; that is $\Delta \Phi_{\text {tot }} \simeq \Delta \Phi$. For example, by considering as two-level system the $5^{2} P_{1 / 2}$ energy splitting between the $F=1$ and $F=2$ levels of ${ }^{87} R b$, and an acceleration of order of $5 \times 10^{16} \mathrm{~m} / \mathrm{s}^{2}$, one has that, in an interferometer with branches of length of $4 \mathrm{~cm}$, the dynamical phase differences $\delta$ can be completely neglected when in such interferometer there is a difference in the arm lengths of about $0.1 \mu \mathrm{m}$.

\section{Quantum Thermometer}

In this section we consider the interaction of an atom with thermal states. A geometric phase identical to the one in (29) appears also in this case. The analysis of the geometric phase (29) in a Mach-Zehnder interferometer could allow very precise measurement of the temperature.

For thermal states, the coefficients $\Sigma_{a}$ and $\Upsilon_{a}$ are replaced by the coefficients $\Sigma_{T}$ and $\Upsilon_{T}$ depending on the temperature [5], $\Sigma_{T}=\left(\gamma_{0} / 4\right)\left(1+4 \pi^{2} k_{B}^{2} T^{2} / \hbar^{2} \omega_{0}^{2}\right)\left(e^{E_{0} / k_{B} T}+1\right) /\left(e^{E_{0} / k_{B} T}-\right.$ $1), E_{0}=\hbar \omega_{0}$, and $\Upsilon_{T}=\left(\gamma_{0} / 4\right)\left(1+4 \pi^{2} k_{B}^{2} T^{2} / \hbar^{2} \omega_{0}^{2}\right)$.

Thus an interferometer in which an atom follows two different paths and interacts with two thermal states at different temperatures can represent a very precise quantum thermometer. Indeed, if the reference temperature of one thermal state is known, the temperature of the other one can be defined by measuring the difference between the geometric phases generated in the two paths.

For example, by measuring $\Delta \Phi_{T}$, one can derive precise estimations of the temperature $T_{c}$ of the colder source, if the temperature $T_{h}$ of the hotter source is known.

We consider the hyperfine structure of atoms and we plot in Figure $2 \Delta \Phi_{T}$ as function of the temperatures of cold sources $T_{c}$, for different ${ }^{133} \mathrm{Cs}$, and ${ }^{87} \mathrm{Rb}$ lines and $T_{h}$ values. In such a figure, the (blue) dashed line is obtained for the energy splitting between the levels $F=4$ and $F=5$ of the $6^{2} P_{3 / 2}$ line of ${ }^{133} \mathrm{Cs}$. In this case $\omega_{0}=251.09 \mathrm{MHz}$ and $\gamma_{0}=$ $32.889 \mathrm{MHz}$ [54] and we considered a reference temperature $T_{h}=10^{-2} \mathrm{~K}$. The (red) dot-dashed line represents $\Delta \Phi_{T}$ for the $F=1 \rightarrow F=2$ transition of the $5^{2} P_{1 / 2}$ line of ${ }^{87} R b$, for which $\omega_{0}=814.5 \mathrm{MHz}$ and $\gamma_{0}=36.129 \mathrm{MHz}$ [53]. A value of $T_{h}=3 \times 10^{-2} \mathrm{~K}$ has been taken into account. We derive the (gray) solid line by considering $T_{h}=6 \times 10^{-2} \mathrm{~K}$ and the splitting between $F=3$ and $F=4$ levels of the $6^{2} P_{1 / 2}$ line

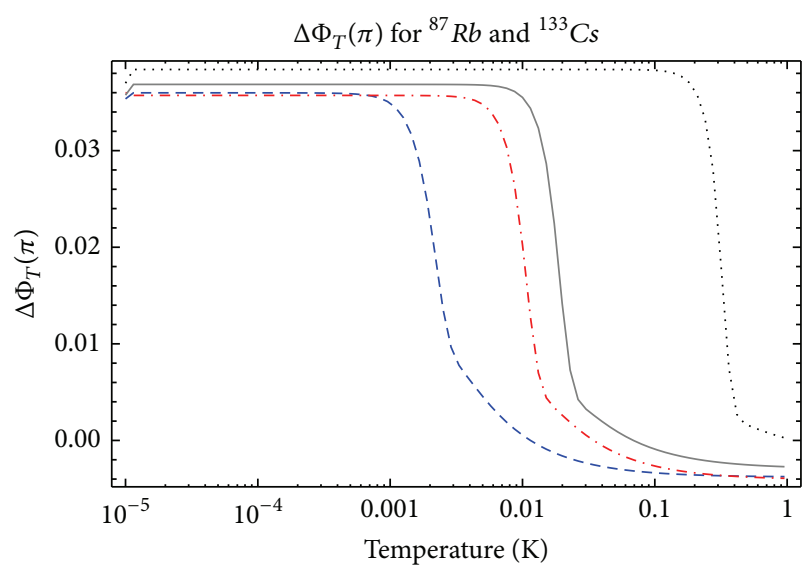

FIgURE 2: Plots of $\Delta \Phi_{T}$ as function of the temperatures of cold sources $T_{c}$, for the splitting between the hyperfine energy levels and $T_{h}$ values: (blue) dashed line: ${ }^{133} \mathrm{Cs}, 6^{2} P_{3 / 2}$ line, splitting for $F=4 \rightarrow$ $F=5$ transition $\left(\omega_{0}=251.09 \mathrm{MHz}, \gamma_{0}=32.889 \mathrm{MHz}\right.$ [54] $)$ and $T_{h}=10^{-2} \mathrm{~K}$; (red) dot-dashed line: ${ }^{87} R b, 5^{2} P_{1 / 2}$ line, splitting for $F=1 \rightarrow F=2$ transition $\left(\omega_{0}=814.5 \mathrm{MHz}, \gamma_{0}=36.129 \mathrm{MHz}\right.$ [53]), and $T_{h}=3 \times 10^{-2} \mathrm{~K}$; (gray) solid line: ${ }^{133} \mathrm{Cs}, 6{ }^{2} P_{1 / 2}$ line, splitting for $F=3 \rightarrow F=4$ transition $\left(\omega_{0}=1167.68 \mathrm{MHz}, \gamma_{0}=\right.$ 28.743 MHz [54]) and $T_{h}=6 \times 10^{-2} \mathrm{~K}$; (black) dotted line: ${ }^{133} \mathrm{Cs}$, $6^{2} S_{1 / 2}$ line, splitting for $F=3 \rightarrow F=4$ transition $\left(\omega_{0}=9.192 \mathrm{GHz}\right.$, $\left.\gamma_{0}=28.743 \mathrm{MHz}[54]\right)$ and $T_{h}=1 \mathrm{~K}$. The time considered is $t \simeq 1 / 4 \omega_{0} \mathrm{~s}$.

of ${ }^{133} \mathrm{Cs}$, for which $\omega_{0}=1167.68 \mathrm{MHz}$ and $\gamma_{0}=28.743 \mathrm{MHz}$ [54]. Moreover, we obtain the (black) dotted line by analyzing the $F=3 \rightarrow F=4$ transition of the $6^{2} S_{1 / 2}$ line of ${ }^{133} C s$. In this case $\omega_{0}=9.192 \mathrm{GHz}, \gamma_{0}=28.743 \mathrm{MHz}$ [54], and $T_{h}=$ $1 \mathrm{~K}$.

The time considered is $t \simeq 1 / 4 \omega_{0} \mathrm{~s}$ in order that the particle decay can be neglected. The result we obtain is that, considering the hyperfine structure of the atoms, one can measure temperatures of the cold source of $\sim 2$ orders of magnitude below the reference temperature of the hot source.

In [47] the Berry phase generated by an atom coupled just to a single mode of a quantum field within a cavity is studied. Here we have studied the role of the geometric phase in the realistic case of the nonunitary evolution of specific atoms interacting with thermal states. Notice that any quantum system interacting with an external field is an open system. Therefore, what is really needed is the analysis of a geometric phase, such as the one in (29), which is defined for mixed states with nonunitary evolution. Moreover, the phase equation (29), contrarily to the Berry phase and its generalization to the mixed state [37], covers the case of noncyclic and nonadiabatic evolution; therefore we are not forced to consider time intervals equal to the period, but we can consider times arbitrarily small, in order to have negligible spontaneous decay in such intervals for the energy level splitting analyzed. Also in this case, paths of slightly different lengths can be chosen in order to let the geometric phase be dominating over the relative dynamical phase.

Our results are thus realistic and new since they refer to specific atoms and their effective nonunitary evolution. 


\section{Conclusions}

We have shown that all the phenomena where vacuum condensates appear generate geometric phases in their time evolution. In particular, we have analyzed the geometric phase for mixed state with a nonunitary evolution for a twolevel atom system. We have shown that atoms with hyperfine structure of the energy levels, as for example ${ }^{87} R b$, accelerated in an interferometer with branches of length of $4 \mathrm{~cm}$ and a difference in the branch lengths of $0.1 \mu \mathrm{m}$ could represent an efficient tool in the laboratory detection of the Unruh effect. On the other hand, we have shown that similar atoms interacting with two different thermal states can be utilized in an interferometer to build a very precise quantum thermometer.

\section{Appendix}

\section{Vacuum Condensate and AAI}

In this Appendix, for the reader's convenience we summarize briefly how the presence of the AAI occurs [5] in all the phenomena in which the vacuum condensate appears [1-12]. For these systems, the physically relevant states $|\Psi(\theta)\rangle,(\theta \equiv \theta(\xi, t)$, with $\xi$ some physically relevant parameter) have indeed nonzero energy variance, $\Delta E(t)=$ $\sqrt{2} \hbar \omega_{\mathbf{k}}\left|U_{\mathbf{k}}(\theta)\right|\left|V_{\mathbf{k}}(\theta)\right|$, and AAI is given by

$$
S(t)=2 \sqrt{2} \int_{0}^{t} \omega_{\mathbf{k}}\left|U_{\mathbf{k}}\left(\theta^{\prime}\right)\right|\left|V_{\mathbf{k}}\left(\theta^{\prime}\right)\right| d t^{\prime},
$$

with $\theta^{\prime} \equiv \theta\left(\xi, t^{\prime}\right)$. Here $U_{\mathbf{k}}(\theta)$ and $V_{\mathbf{k}}(\theta)$ are the Bogoliubov coefficients entering in the transformation $|\Psi(\theta)\rangle=$ $J^{-1}(\theta)|\psi(t)\rangle$, with $|\psi(t)\rangle$ original state and $J^{-1}(\theta)$ generator of the Bogoliubov transformation:

$$
\begin{aligned}
\alpha_{\mathbf{k}}^{r}(\theta) & =J^{-1}(\theta) a_{\mathbf{k}}^{r}(t) J(\theta) \\
& =U_{\mathbf{k}}(\theta) a_{\mathbf{k}}^{r}(t)+V_{\mathbf{k}}(\theta) a_{-\mathbf{k}}^{r \dagger}(t)
\end{aligned}
$$

$U_{\mathbf{k}}$ and $V_{\mathbf{k}}$ depend on the system one considers and satisfy the relation $\left|U_{\mathbf{k}}\right|^{2} \pm\left|V_{\mathbf{k}}\right|^{2}=1$, with + for fermions and - for bosons.

Notice that the vacuum state $|0(\theta)\rangle$ for such systems is related to the original one $|0\rangle$ by the relation, $|0(\theta)\rangle=$ $J^{-1}(\theta)|0\rangle$. Therefore, (1) shows that all the phenomena characterized by the presence of modifications of vacuum fluctuations (which are all described by Bogoliubov transformations) are also characterized by the presence of the geometric phase in their evolution.

For example, in the case of the Unruh effect, the Bogoliubov coefficients that allow to express the Minkowski vacuum in terms of Rindler states are for bosons $U_{\mathbf{k}}=$ $\sqrt{e^{2 \pi \omega_{\mathbf{k}} / a} /\left(e^{2 \pi \omega_{\mathbf{k}} / a}-1\right)}$ and $V_{\mathbf{k}}=\sqrt{1 /\left(e^{2 \pi \omega_{\mathbf{k}} / a}-1\right)}$ and similar for fermions. Here $a$ is the acceleration of the observer. The relation between the Minkowski $|0\rangle_{M}$ and Rindler $|0\rangle_{R}$ vacua in the case of a single scalar field [55] is

$$
|0\rangle_{M} \sim \exp \left(\frac{1}{2} \sum_{\mathbf{k}} e^{-\pi \omega_{\mathbf{k}} / a} a_{R}^{\dagger} a_{L}^{\dagger}\right)|0\rangle_{R},
$$

where $R$ and $L$ refer to modes supported in the right and left Rindler wedges, respectively.

\section{Conflict of Interests}

The authors declare that there is no conflict of interests regarding the publication of this paper.

\section{Acknowledgment}

Partial financial support from MIUR and INFN is acknowledged.

\section{References}

[1] W. G. Unruh, "Notes on black-hole evaporation," Physical Review D, vol. 14, no. 4, pp. 870-892, 1976.

[2] S. W. Hawking, "Particle creation by black holes," Communications in Mathematical Physics, vol. 43, no. 3, pp. 199-220, 1975, Erratum in: Communications in Mathematical Physics, vol. 46, p. 206, 1976.

[3] L. Parker, "Particle creation in expanding universes," Physical Review Letters, vol. 21, no. 8, pp. 562-564, 1968.

[4] E. Schrödinger, "The proper vibrations of the expanding universe," Physica, vol. 6, no. 7-12, pp. 899-912, 1939.

[5] A. Capolupo and G. Vitiello, "Probing Hawking and Unruh effects and quantum field theory in curved space by geometric invariants," Physical Review D, vol. 88, no. 2, Article ID 024027, 9 pages, 2013.

[6] H. Umezawa, Advanced Field Theory: Micro, Macro, and Thermal Physics, AIP, New York, NY, USA, 1993.

[7] M. Blasone, A. Capolupo, and G. Vitiello, "Quantum field theory of three flavor neutrino mixing and oscillations with CP violation," Physical Review D, vol. 66, no. 2, Article ID 025033, 2002.

[8] M. Blasone, A. Capolupo, O. Romei, and G. Vitiello, "Quantum field theory of boson mixing," Physical Review D, vol. 63, no. 12, Article ID 125015, 2001.

[9] A. Capolupo, S. Capozziello, and G. Vitiello, "Dark energy and particle mixing," Physics Letters A, vol. 373, no. 6, pp. 601-610, 2009.

[10] M. Blasone, A. Capolupo, S. Capozziello, S. Carloni, and G. Vitiello, "Neutrino mixing contribution to the cosmological constant," Physics Letters A, vol. 323, no. 3-4, pp. 182-189, 2004.

[11] A. Capolupo and M. Di Mauro, "Spontaneous supersymmetry breaking induced by vacuum condensates," Physics Letters A, vol. 376, no. 45, pp. 2830-2833, 2012.

[12] N. D. Birrell and P. C. W. Davies, Quantum Fields in Curved Space, Cambridge Monographs on Mathematical Physics, Cambridge University Press, Cambridge, UK, 1984.

[13] J. Anandan and Y. Aharonov, "Geometry of quantum evolution," Physical Review Letters, vol. 65, no. 14, pp. 1697-1700, 1990.

[14] A. K. Pati, "Relation between 'phases' and 'distance' in quantum evolution,” Physics Letters A, vol. 159, no. 3, pp. 105-112, 1991.

[15] A. K. Pati, "New derivation of the geometric phase," Physics Letters A, vol. 202, no. 1, pp. 40-45, 1995.

[16] M. V. Berry, "Quantal phase factors accompanying adiabatic changes," Proceedings of the Royal Society of London Series A, Mathematical and Physical Sciences, vol. 392, no. 1802, pp. 4557, 1984. 
[17] Y. Aharonov and J. Anandan, "Phase change during a cyclic quantum evolution," Physical Review Letters, vol. 58, no. 16, pp. 1593-1596, 1987.

[18] J. Samuel and R. Bhandari, "General setting for Berry's phase," Physical Review Letters, vol. 60, article 2339, 1988.

[19] N. Mukunda and R. Simon, "Quantum kinematic approach to the geometric phase. I. General formalism," Annals of Physics, vol. 228, no. 2, pp. 205-268, 1993.

[20] G. Garcia de Polavieja, "Noncyclic geometric phase shift for quantal revivals," Physical Review Letters, vol. 81, no. 1, pp. 1-5, 1998.

[21] S. Pancharatnam, "Generalized theory of interference, and its applications, part 1. Coherent pencil," Proceedings of the Indian Academy of Sciences A, vol. 44, pp. 247-262, 1956.

[22] A. Shapere and F. Wilczek, Geometric Phases in Physics, World Scientific, Singapore, 1989.

[23] B. Simon, "Holonomy, the quantum adiabatic theorem, and Berry's phase," Physical Review Letters, vol. 51, no. 24, pp. 21672170, 1983.

[24] J. C. Garrison and E. M. Wright, "Complex geometrical phases for dissipative systems," Physics Letters A, vol. 128, no. 3-4, pp. 177-181, 1988.

[25] J. C. Garrison and R. Y. Chiao, "Geometrical phases from global gauge invariance of nonlinear classical field theories," Physical Review Letters, vol. 60, no. 3, pp. 165-168, 1988.

[26] A. K. Pati, "Gauge-invariant reference section and geometric phase," Journal of Physics A, vol. 28, no. 7, pp. 2087-2094, 1995.

[27] A. K. Pati, "Geometric aspects of noncyclic quantum evolutions," Physical Review A, vol. 52, no. 4, pp. 2576-2584, 1995.

[28] J. Anandan, "Nonadiabatic nonabelian geometric phase," Physics Letters A, vol. 133, no. 4-5, pp. 171-175, 1988.

[29] A. Mostafazadeh, "Noncyclic geometric phase and its nonabelian generalization," Journal of Physics A: Mathematical and General, vol. 32, no. 46, pp. 8157-8171, 1999.

[30] Z. S. Wang and Q. Liu, "Geometric phase and spinorial representation of mixed state," Physics Letters A, vol. 377, no. 45-48, pp. 3272-3278, 2013.

[31] A. Uhlmann, "Parallel transport and 'quantum holonomy' along density operators," Reports on Mathematical Physics, vol. 24, no. 2, pp. 229-240, 1986.

[32] E. Sjöqvist, A. K. Pati, A. Ekert et al., "Geometric phases for mixed states in interferometry," Physical Review Letters, vol. 85, no. 14, pp. 2845-2849, 2000.

[33] A. Carollo, I. Fuentes-Guridi, M. F. Santos, and V. Vedral, "Geometric phase in open systems," Physical Review Letters, vol. 90, Article ID 160402, 2003.

[34] Z. S. Wang, L. C. Kwek, C. H. Lai, and C. H. Oh, "Geometric phase in open two-level system," Europhysics Letters, vol. 74, no. 6, pp. 958-964, 2006.

[35] S. Chaturvedi, E. Ercolessi, G. Marmo, G. Morandi, N. Mukunda, and R. Simon, "Geometric phase for mixed states: a differential geometric approach," The European Physical Journal C: Particles and Fields, vol. 35, no. 3, pp. 413-423, 2004.

[36] M. Ericsson, A. K. Pati, E. Sjöqvist, J. Brännlund, and D. K. L. Oi, "Mixed state geometric phases, entangled systems, and local unitary transformations," Physical Review Letters, vol. 91, no. 9, Article ID 090405, 2003.

[37] D. M. Tong, E. Sjöqvist, L. C. Kwek, and C. H. Oh, "Kinematic approach to the mixed state geometric phase in nonunitary evolution," Physical Review Letters, vol. 93, Article ID 080405, 2004.
[38] A. Tomita and R. Y. Chiao, “Observation of Berry's topological phase by use of an optical fiber," Physical Review Letters, vol. 57, no. 8, pp. 937-940, 1986.

[39] J. A. Jones, V. Vedral, A. Ekert, and G. Castagnoll, "Geometric quantum computation using nuclear magnetic resonance," Nature, vol. 403, no. 6772, pp. 869-871, 2000.

[40] P. J. Leek, J. M. Fink, A. Blais et al., “Observation of Berry's phase in a solid-state qubit," Science, vol. 318, no. 5858, pp. 1889-1892, 2007.

[41] M. Neeley, M. Ansmann, R. C. Bialczak et al., "Emulation of a quantum spin with a superconducting phase qudit," Science, vol. 325, no. 5941, pp. 722-725, 2009.

[42] M. Pechal, S. Berger, A. A. Abdumalikov et al., "Geometric phase and nonadiabatic effects in an electronic harmonic oscillator," Physical Review Letters, vol. 108, no. 17, Article ID 170401, 2012.

[43] A. Capolupo, "Probing CPT violation in meson mixing by a noncyclic phase," Physical Review D, vol. 84, Article ID 116002, 2011.

[44] A. Capolupo and G. Vitiello, "Spontaneous supersymmetry breaking probed by geometric invariants," Advances in High Energy Physics, vol. 2013, Article ID 850395, 5 pages, 2013.

[45] J. Hu and H. Yu, "Geometric phase for an accelerated two-level atom and the Unruh effect," Physical Review A, vol. 85, Article ID 032105, 2012.

[46] E. Martín-Martínez, I. Fuentes, and R. B. Mann, "Using berry’s phase to detect the Unruh effect at lower accelerations," Physical Review Letters, vol. 107, Article ID 131301, 2011.

[47] E. Martín-Martínez, A. Dragan, R. B. Mann, and I. Fuentes, "Berry phase quantum thermometer," New Journal of Physics, vol. 15, Article ID 053036, 2013.

[48] C. Sabín, A. White, L. Hackermuller, and I. Fuentes, "Impurities as a quantum thermometer for a Bose-Einstein condensate," Scientific Reports, vol. 4, article 6436, 2014.

[49] G. Compagno, R. Passante, and F. Persico, Atom-Field Interactions and Dressed Atoms, Cambridge University Press, Cambridge, UK, 1995.

[50] G. Lindblad, "On the generators of quantum dynamical semigroups," Communications in Mathematical Physics, vol. 48, no. 2, pp. 119-130, 1976.

[51] V. Gorini, A. Kossakowski, and E. C. Sudarshan, "Completely positive dynamical semigroups of $N$-level systems," Journal of Mathematical Physics, vol. 17, no. 5, p. 821, 1976.

[52] D. A. Steck, "Rubidium 85 D Line Data," revision 2.1.6, 2013, http://steck.us/alkalidata.

[53] D. A. Steck, "Rubidium 87 D Line Data," revision 2.1.4, 2010, http://steck.us/alkalidata.

[54] D. A. Steck, Cesium D Line Data, revision 2.1.4, 2010, http://steck .us/alkalidata.

[55] L. C. B. Crispino, A. Higuchi, and G. E. A. Matsas, "The Unruh effect and its applications," Reviews of Modern Physics, vol. 80, no. 3, pp. 787-838, 2008. 

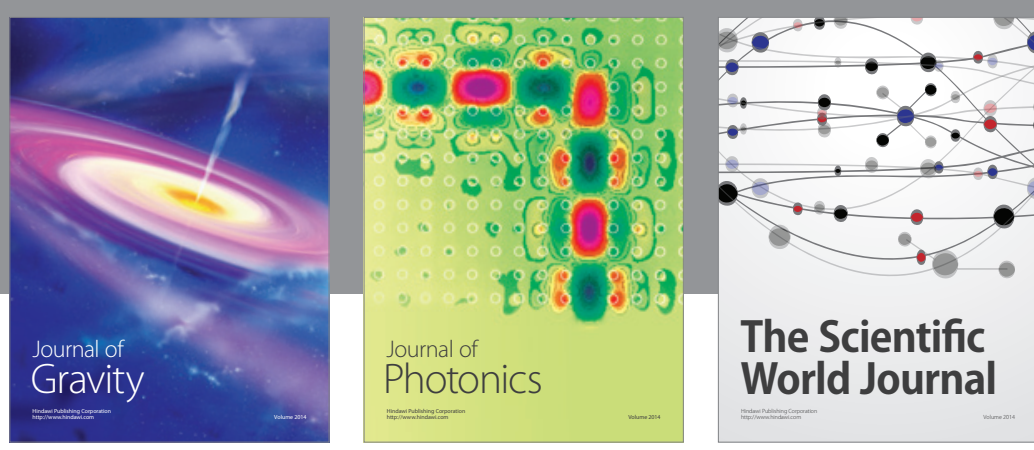

The Scientific World Journal
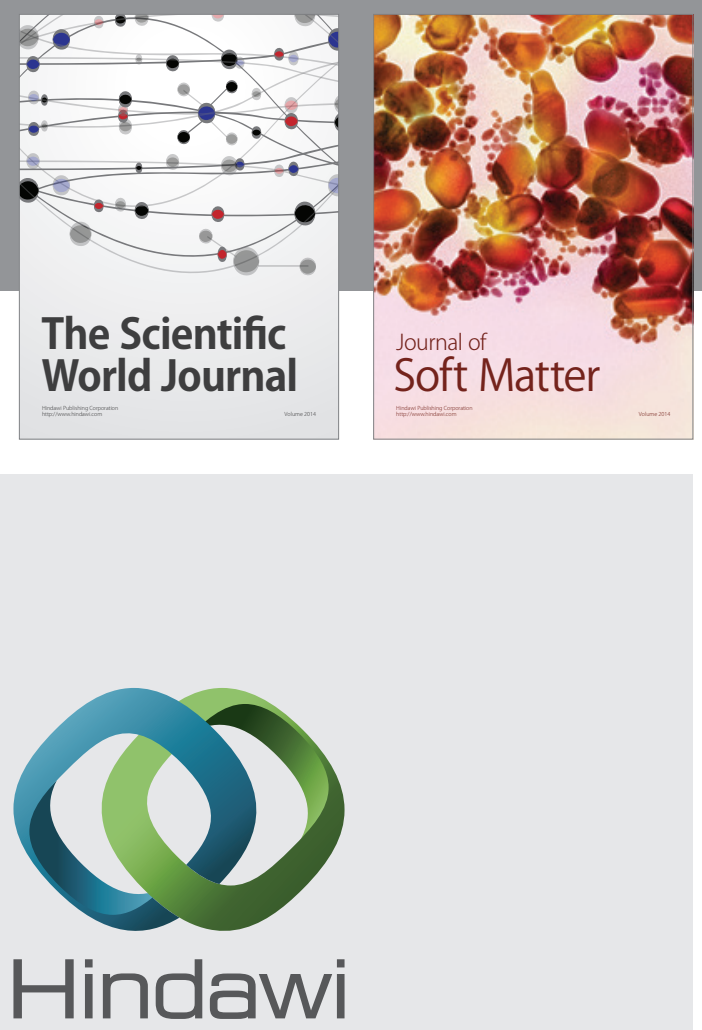

Submit your manuscripts at

http://www.hindawi.com

nternational Journal of

Statistical Mechanics
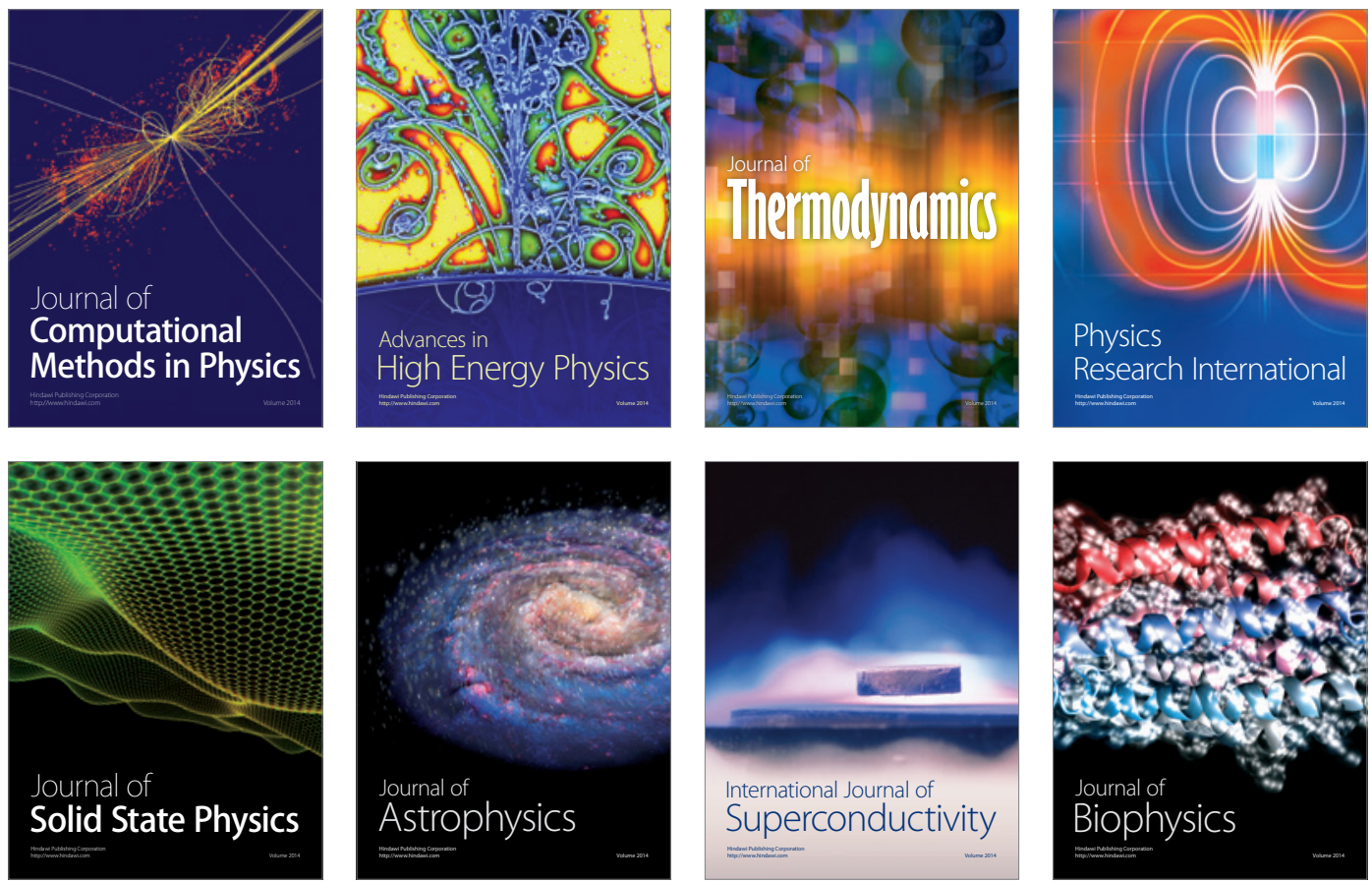
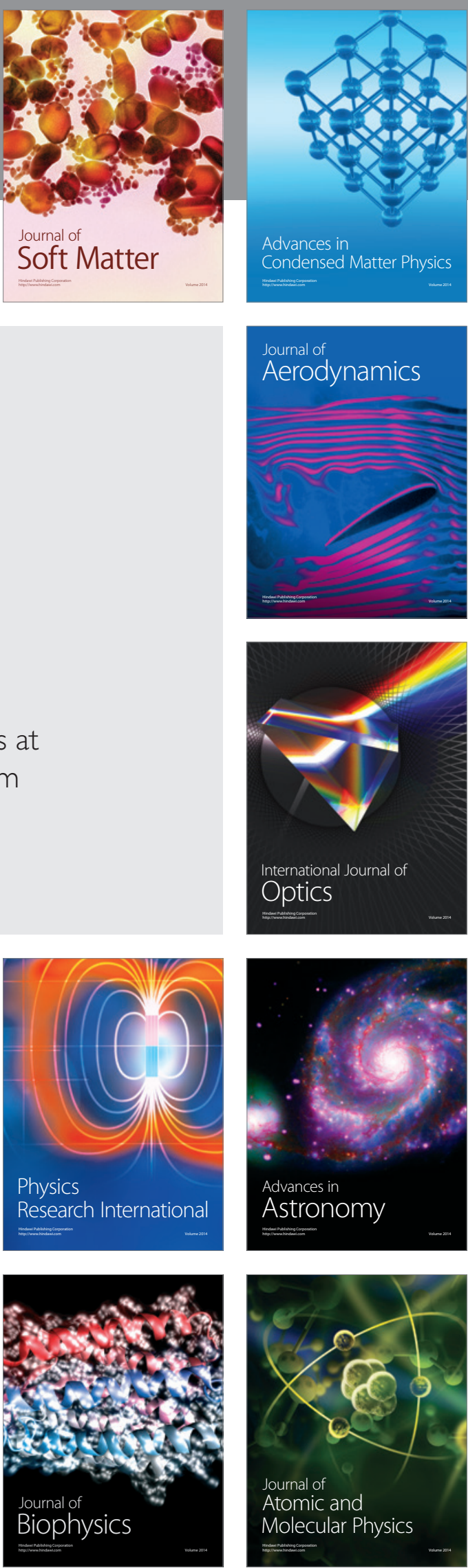